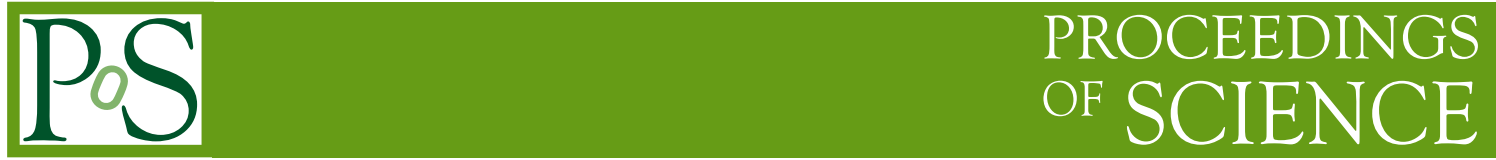

\title{
Review of analysis methods for correlations and fluctuations
}

Thomas A. Trainor* and Duncan J. Prindle

University of Washington

E-mail: trainor@hausdorf.npl.washington.edu,

prindleenpl.washington.edu

\begin{abstract}
We review fluctuation and correlation analysis methods at the SPS and RHIC. We identify basic issues of fluctuation measure design. We show that fluctuation scale dependence is related to angular autocorrelations by an integral equation. We discuss the optimum projection of twoparticle momentum space to 2D subspaces with minimal distortion. We list mechanisms currently known to produce number and $p_{t}$ correlations. We conclude with comments on several current measure designs.
\end{abstract}

Correlations and Fluctuations in Relativistic Nuclear Collisions

July 7-9 2006

Florence, Italy

\footnotetext{
* Speaker.
} 


\section{Introduction}

The terms fluctuations and correlations applied to nuclear collisions refer to significant eventwise changes in the structure of particle distributions on single-particle and two-particle momentum space. Fluctuation measurements at a single bin size or scale (e.g., detector acceptance) could arise from many configurations of the multiparticle momentum distribution. In contrast, the scale dependence of fluctuations over a significant scale interval provides unique information about twoparticle correlation structure. Those aspects of distributions on $(\eta, \phi)$ depending on angle differences are retained in the form of angular autocorrelations [1]. Complementary to angular autocorrelations are two-particle correlations on transverse momentum $p_{t}$ or rapidity $y_{t}$.

Much experience has been gained from a sequence of fluctuation and correlation measurements at the SPS and RHIC. Recent results have alerted us to several new physics issues not anticipated in earlier measure design. We have learned that autocorrelations can be extracted from fluctuation scale dependence with numerical inversion techniques [2]. We now have a better idea of the general structure of momentum space for different collision systems. We've reached a stage where a consolidation of measurement techniques and interpretations is possible and necessary.

In this paper we present elements of a unified system of fluctuation/correlation measurement in a historical context. We first review existing analysis methods. We then describe two basic statistical elements: fluctuations on a binned momentum space and Pearson's normalized covariance. We define scale-dependent normalized variances and variance differences. We relate fluctuation scale dependence to angular autocorrelations through an integral equation. Numerical solution of the integral equation reduces fluctuation scale dependence to angular correlations. We propose an optimum projection of the 6D two-particle momentum space to 2D subspaces and discuss two main correlation types. We summarize the current physics of $n$ and $p_{t}$ correlations and conclude with some case studies of measure design which illustrate the importance of proper design criteria.

\section{A Multiplicity of Methods}

Over the past decade a number of fluctuation measures has been introduced to the SPS and RHIC heavy ion programs. Some of them share a few common features: 1) a global random variable is defined based on a physics hypothesis (e.g., by analogy with a thermodynamic variable), 2) the variance of the global variable about its mean is defined, 3) the variance is compared to a 'statistical' reference to obtain a measure of excess or 'nonstatistical' fluctuations. Global variables are defined as sums, differences and ratios of elementary random variables (e.g., $p_{t}, n, n_{+}, n_{-}, n_{\pi}$, $n_{K}$ ). Comparison of a variance with its reference is accomplished via differences and/or ratios.

The $p_{t}$ fluctuation measures deliberately designed to test a linear superposition hypothesis are $\Phi_{p_{t}}=\sqrt{\frac{\overline{\left(p_{t}-n \hat{p}_{t}\right)^{2}}}{\bar{n}}}-\sigma_{\hat{p}_{t}}[3,4]$ and the closely-related $\Delta \sigma_{p_{t}: n}^{2}=\frac{\overline{\left(p_{t}-n \hat{p}_{t}\right)^{2}}}{\bar{n}}-\sigma_{\hat{p}_{t}}^{2}$ [5]. They compare a per-particle normalized variance to its central-limit value. Some measures of $\left\langle p_{t}\right\rangle$ fluctuations such as $\sigma_{p_{t}, \text { dynamical }}^{2}=\sigma_{\left\langle p_{t}\right\rangle}^{2}-\sigma_{\hat{p}_{t}}^{2} / \bar{n}[6]$ and $F_{p_{t}}=\sqrt{\bar{n} \sigma_{\left\langle p_{t}\right\rangle}^{2} / \sigma_{\hat{p}_{t}}^{2}}-1$ [7] are biased (the estimate systematically deviates from the intended parent property) for small multiplicities $n$. The bias is very large and arises from properties of $\sigma_{\left\langle p_{t}\right\rangle}^{2}$, a variance of the ratio of two random variables ( $c f$. Sec. 11 for details). $\left\langle\delta p_{t} \cdot \delta p_{t}\right\rangle \equiv \overline{\left\{\frac{\sum_{i \neq j}\left(p_{t i}-\hat{p}_{t}\right)\left(p_{t j}-\hat{p}_{t}\right)}{n(n-1)}\right\}}[8]$, with $\sum_{i=1}^{n}\left(p_{t i}-\hat{p}_{t}\right)=p_{t}-n \hat{p}_{t}$, is inter- 
mediate between a fluctuation (single-particle counting) and correlation (pair counting) measure. The principal bias in that definition involves a cross-contamination (covariance) between $p_{t}$ and $n$ fluctuations ( $c f$. Sec. 11).

$\Sigma_{p_{t}, S T A R}=\sqrt{\sigma_{p_{t}, \text { dynamical }}^{2} / \hat{p}_{t}^{2}}[8]$ and $\Sigma_{p_{t}, \text { CERES }}=\sqrt{\Delta \sigma_{p_{t}: n}^{2} / \bar{n} \hat{p}_{t}^{2}}[9]$ are motivated by a specific collision model which assumes that each A-A collision is fully thermalized. Excess $\left\langle p_{t}\right\rangle$ fluctuations should then arise only because of event-wise variation of global temperature $T$. In that model $\sigma_{p_{t}, \text { dynamical }}^{2}$ is taken as the analog of temperature variance $\sigma_{T}^{2}$, the analog for ensemble-mean $T_{0}$ is assumed to be $\hat{p}_{t}$ (ensemble-mean particle $p_{t}$ ), and $\Sigma_{p_{t}} \rightarrow \delta T / T_{0}$. If event-wise thermalization is not achieved (there is plentiful evidence to that effect), those model-dependent measures are difficult to interpret [10].

We encounter a similar assortment of measures for number fluctuations. For multiplicity fluctuations there is the normalized variance $\sigma_{n}^{2} / \bar{n}$ with $\sigma_{n}^{2}=\overline{(n-\bar{n})^{2}}$. For net charge $Q \equiv n_{+}-n_{-}$ there is $\sigma_{Q}^{2} / \bar{n}$. Both have central-limit reference values 1 , so suitable differential fluctuation measures which test a linear superposition hypothesis are $\Delta \sigma_{n /}^{2}=\sigma_{n}^{2} / \bar{n}-1$ for $n$ and the equivalent for $Q$. Those measures are directly related to two-particle number autocorrelations. Net-charge fluctuation measure $D[11]$ was defined in terms of ratio $n_{+} / n_{-}$, and is consequently strongly biased (that measure first revealed the bias arising from ratios). $v_{\text {dynamical }}$, defined in terms of $n_{+} / \bar{n}_{+}-n_{-} / \bar{n}_{-}$, is described as a 'robust' measure because it is said to cancel particle detection efficiencies in the ratios [12]. That claim is questionable because relevant single-particle and pair efficiencies are generally different. The $p_{t}$ and $n$ fluctuation measures subscripted 'dynamical' include a trivial factor $1 / n$ which adds to interpretation difficulty.

Given the large array of fluctuation measures is there a smaller optimum set, are there clear design criteria? The answer is yes, but resolution of design ambiguities requires understanding the basics of fluctuation measurement, the relation between fluctuations and correlations and the ultimate goal of two-particle correlation analysis: projection of 6D two-particle momentum space to viewable 2D subspaces with minimal information loss or distortion. The result should be accurate and model-independent characterizations of correlation structure in nuclear collisions.

\section{Fluctuation Basics}

The data system for any fluctuation or correlation analysis is an ensemble of particle distributions (events) on single-particle momentum space $\left(p_{t}, \eta, \phi\right)$ or $\left(y_{t}, \eta, \phi\right)$, where $p_{t}$ is transverse momentum, $m_{t}$ is transverse mass, $\eta$ is pseudorapidity, $\phi$ is azimuth and $y_{t} \equiv \ln \left\{\left(m_{t}+p_{t}\right) / m_{0}\right\}$ is transverse rapidity, with the pion mass assigned to $m_{0}$ for unidentified hadrons. Momentum space is bounded by a detector acceptance. The space within the acceptance is binned according to a range of bin sizes. Transverse momentum $p_{t}$ can be considered a continuous measure distributed on subspace $(\eta, \phi)$ and sampled by individual particles in each event.

An ensemble of distributions (events) on $x$ is sketched in Fig. 1 (first panel). Each event is compared with an ensemble-average reference distribution to determine relative information, measured by fluctuations of bin contents about their means. Event-wise pair distributions on space $\left(x_{1}, x_{2}\right)$ in the second panel are compared to a reference formed from mixed pairs or a 2D (Cartesian) product of single-particle ensemble-average distributions. The differences reveal correlations in the two-particle distribution corresponding to fluctuations in the single-particle distribution [13]. 
That relation is the basis for the integral equation relating fluctuations to correlations described in Sec. 6 .
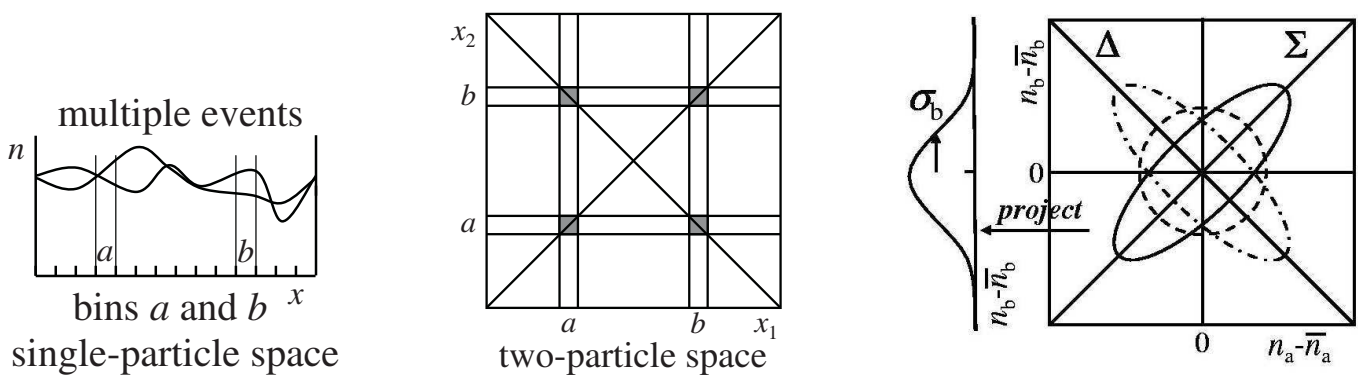

Figure 1: Event-wise distributions on a binned primary space, corresponding two-particle space and possible distributions of bin contents for selected bins $a$ and $b$ in the primary space.

The basic random variables are bin contents $p_{t}$ and $n$ (or $n_{+}, n_{-}$, etc.) in pairs of bins $a$ and $b$ combined as products: $n_{a}^{2}, n_{b}^{2}$ or $n_{a} n_{b}$. The ensemble averages of the products are compared to the products of averages (the reference). Their linear combinations are the variances $\sigma_{a, b}^{2}$ (diagonal bins in the center panel) and covariances $\sigma_{a b}^{2}$ (off-diagonal bins). We are especially interested in how variances (fluctuations) change with bin size or scale, and therefore how they relate to covariances (two-particle correlations).

Fig. 1 (third panel) sketches possible frequency distributions on $\left(n_{a}, n_{b}\right)$ from bin pair $(a, b)$ in the first panel. The ellipses represent half-maximum contours for gaussian-random fluctuations. The three cases correspond to correlation (solid curve), anticorrelation (dash-dot curve) and no correlation (dashed curve) between bins $a$ and $b$, the last being expected for a mixed-pair reference. The 2D frequency distribution is characterized statistically by two marginal (projection) variances and a covariance. The marginal variances for bins $a$ and $b$ are $\sigma_{a, b}^{2}=\overline{(n-\bar{n})_{a, b}^{2}}=\overline{n_{a, b}^{2}}-\bar{n}_{a, b}^{2}$, and the covariance is $\sigma_{a b}^{2}=\overline{(n-\bar{n})_{a}(n-\bar{n})_{b}}=\overline{n_{a} n_{b}}-\bar{n}_{a} \bar{n}_{b}$. From those basic quantities we can define a relative covariance, the basis for statistical measures which test a linear-superposition hypothesis.

\section{Pearson's normalized covariance}

Pearson's correlation coefficient is a measure of relative or normalized covariance [14]. For number fluctuations in bin pair $(a, b)$, as sketched in Fig. 1 (right panel), the normalized covariance is defined by

$$
r_{a b} \equiv \frac{\sigma_{a b}^{2}}{\sqrt{\sigma_{a}^{2} \sigma_{b}^{2}}} \in[-1,1]
$$

The numerator is the covariance for bin pair $(a, b)$, and the denominator is the geometric mean of $a$ and $b$ marginal variances. The distribution of $r_{a b}$ for histogram bin pairs in Fig. 1 (second panel) fully represents two-particle correlations on space $x$. Fig. 2 (first three panels) illustrates limiting cases of Fig. 1, (third panel), with the corresponding values of $r_{a b}$.

Tests of linear superposition include the normalized covariance in the following way. Suppose a fluctuating system (larger bin) is separated into two parts (equivalent smaller bins) $a$ and $b$. We 


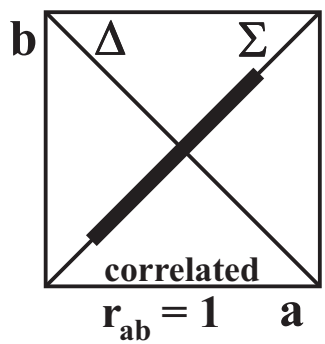

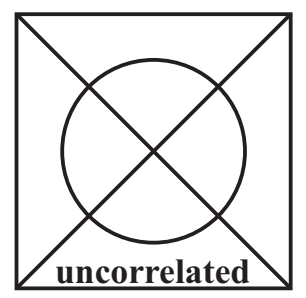

$\mathbf{0}$

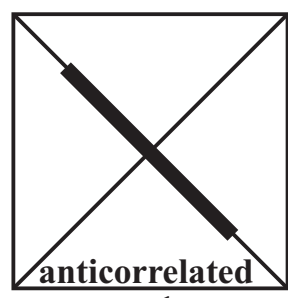

$-1$

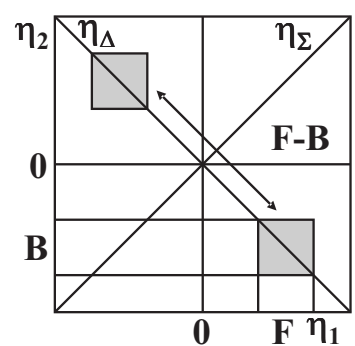

Figure 2: Examples of Pearson's coefficient and F-B correlations.

want to determine statistically whether the total system is a linear superposition of $a$ and $b$. It is easy to show that for number variances and covariance

$$
\sigma_{a+b}^{2}=\sigma_{a}^{2}+\sigma_{b}^{2}+2 \sigma_{a b}^{2}
$$

For $n=n_{a}+n_{b}$ we have

$$
\frac{\sigma_{n}^{2}}{\bar{n}}=\frac{\bar{n}_{a}}{\bar{n}} \frac{\sigma_{a}^{2}}{\bar{n}_{a}}+\frac{\bar{n}_{b}}{\bar{n}} \frac{\sigma_{b}^{2}}{\bar{n}_{b}}+\frac{\sqrt{\bar{n}_{a} \bar{n}_{b}}}{\bar{n}} \frac{\sigma_{a b}^{2}}{\sqrt{\bar{n}_{a} \bar{n}_{b}}}
$$

The normalized variance of the whole is a weighted mean of variances from the parts and a covariance. Linear superposition means that covariance $\sigma_{a b}^{2}$ between subsystems $a$ and $b$ is zero (central limit theorem). In that case a normalized variance is independent of composition (independent of scale or bin size): $\sigma_{n}^{2} / \bar{n}=\sigma_{a}^{2} / \bar{n}_{a}=\sigma_{b}^{2} / \bar{b}$ [13]. Deviations from linear superposition (i.e., interesting physics) are revealed by a nonzero $\sigma_{a b}^{2} / \sqrt{\bar{n}_{a} \bar{n}_{b}}$ distribution, or equivalently by the scale dependence of $\sigma_{n}^{2} / \bar{n}[13]$.

Given those results and other relations in the next section we adopt the following definitions for the $n$ and $p_{t}$ normalized covariances

$$
r_{a b} \equiv \frac{\sigma_{a b}^{2}}{\sqrt{\sigma_{a}^{2} \sigma_{b}^{2}}} \rightarrow \frac{\overline{(n-\bar{n})_{a}(n-\bar{n})_{b}}}{\sqrt{\bar{n}_{a} \bar{n}_{b}}} \text { and } \frac{\overline{\left(p_{t}-n \hat{p}_{t}\right)_{a}\left(p_{t}-n \hat{p}_{t}\right)_{b}}}{\sqrt{\bar{n}_{a} \bar{n}_{b}}}
$$

used in our analysis. The quantities on the right are modified from the Pearson definition. They include in the denominators not the marginal variances but the central-limit or Poisson values of those variances. We also omit the central-limit $\sigma_{\hat{p}_{t}}^{2}$ factor from the denominator of the $p_{t}$ normalized covariance for reasons discussed in Sec. 5.

The last panel of Fig. 2 illustrates so-called forward-backward (FB) correlations [15], in which correlations on pseudorapidity $\eta$ are measured by $F B=\overline{(n-\bar{n})_{\mathrm{F}} \cdot(n-\bar{n})_{\mathrm{B}}} / \sqrt{\sigma_{n(\mathrm{~F})}^{2} \cdot \sigma_{n(\mathrm{~B})}^{2}}$, an example of Pearson's normalized covariance. Covariance $F B$ is simply related to the $2 \mathrm{D}$ angular autocorrelation projected onto difference axis $\eta_{\Delta}=\eta_{1}-\eta_{2}$. Fig. 2 (fourth panel) should be compared to Fig. 1 (second panel) and Fig. 4 (third panel).

\section{Scale-dependent differential fluctuation measures}

In this section we combine several related elements to obtain a self-consistent fluctuation measure system. We combine the closely-related concepts of scale invariance of the total variance as a manifestation of the central limit theorem (CLT) and Pearson's normalized covariance as a test of linear superposition. 


\subsection{Total variance}

In a detailed study of $\Phi_{p_{t}}$ and its relation to the central limit theorem and linear superposition we introduced the concept of the total $p_{t}$ variance $\Sigma_{p_{t}: n}^{2}$ and total variance difference $\Delta \Sigma_{p_{t}: n}^{2}=$ $\Sigma_{p_{t}: n}^{2}-\Sigma_{p_{t}, \text { ref }}^{2}[13]$. The total variance difference is defined by

$$
\Delta \Sigma_{p_{t}: n}^{2} \equiv \overline{\sum_{i \neq j=1}^{n, n-1}\left(p_{t i}-\hat{p}_{t}\right)\left(p_{t j}-\hat{p}_{t}\right)}=\overline{\sum_{i, j=1}^{n}\left(p_{t i}-\hat{p}_{t}\right)\left(p_{t j}-\hat{p}_{t}\right)}-\overline{\sum_{i=1}^{n}\left(p_{t i}-\hat{p}_{t}\right)^{2}}
$$

with $\sum_{i=1}^{n}\left(p_{t i}-\hat{p}_{t}\right)=p_{t}-n \hat{p}_{t}$ summed over a bin. On the left is is an integral over two-particle momentum space (sum of pairs). On the right is a differential fluctuation measure based on singleparticle counting. Dividing through by the mean bin multiplicity we have

$$
\frac{\Delta \Sigma_{p_{t}: n}^{2}}{\bar{n}}=\Delta \sigma_{p_{t}: n}^{2}=\frac{\overline{\left(p_{t}-n \hat{p}_{t}\right)^{2}}}{\bar{n}}-\sigma_{\hat{p}_{t}}^{2}
$$

The total variance as an integral over a two-particle space is the basis for the integral equation which relates fluctuation scale dependence to angular autocorrelations ( $c f$. Sec. 6) [2].

\subsection{Variance differences}

If $b \rightarrow a$ in the normalized covariance (diagonal bins in Fig. 1, second panel) we obtain the normalized variance for bin $a: r_{a a}=\overline{(n-\bar{n})_{a}^{2}} / \bar{n}_{a}$. The average over all bins in an acceptance is then the normalized number variance $\sigma_{n /}^{2} \equiv \sigma_{n}^{2} / \bar{n}=\overline{(n-\bar{n})^{2}} / \bar{n}$, also referred to as a 'scaled' variance (we reserve 'scale' to mean bin size). We now define a compatible set of normalized variances, covariances and variance differences which provide optimal measurement of fluctuation scale dependence and two-particle correlations in nuclear collisions. To establish exact connections among all statistical measures for $p_{t}$ and $n$ we first introduce a decomposition of the ordinary $p_{t}$ variance by adding and subtracting $n \hat{p}_{t}$ within the LHS quadratic

$$
\overline{\left(p_{t}-\bar{p}_{t}\right)^{2}}=\overline{\left(p_{t}-n \hat{p}_{t}\right)^{2}}+2 \hat{p}_{t} \overline{\left(p_{t}-n \hat{p}_{t}\right) \cdot(n-\bar{n})}+\hat{p}_{t}^{2} \overline{(n-\bar{n})^{2}}
$$

Dividing through by $\bar{n}$ we obtain on the RHS two normalized variances and a normalized covariance

$$
\sigma_{p_{t}: n}^{2} \equiv \frac{\overline{\left(p_{t}-n \hat{p}_{t}\right)^{2}}}{\bar{n}} \quad \sigma_{p_{t}: n-n}^{2} \equiv \frac{\overline{\left(p_{t}-n \hat{p}_{t}\right) \cdot(n-\bar{n})}}{\bar{n}} \quad \sigma_{n /}^{2} \equiv \frac{\overline{(n-\bar{n})^{2}}}{\bar{n}}
$$

The first item is the normalized conditional $p_{t}$ variance. Subscript $p_{t}: n$ reads ' $p_{t}$ fluctuations given $n$.' The second item is the normalized covariance between $p_{t}$ fluctuations given $n$ and number fluctuations. ${ }^{1}$ The last term is the normalized number variance. The normalized variances are scale-dependent (scale $=$ bin size $\delta x$ ), and when expressed in terms of bin sums $p_{t}(\delta x)$ and $n(\delta x)$ are running integrals of normalized covariance densities.

To complete the correspondence between fluctuation scale dependence and correlations we define a 'zero-scale limit' to the scale-dependent fluctuation measures. The limit coincides with the central-limit reference for each quantity—independent samples from a fixed parent—since in

\footnotetext{
${ }^{1}$ That covariance was the initial motivation for $\Phi_{p_{t}}[3]$.
} 
the zero-scale limit $(\delta x \rightarrow 0)$ each occupied bin contains at most one particle (independent samples) and two-particle correlations play no role. The CLT references for the three Eqs. (5.4) are respectively $\sigma_{\hat{p}_{t}}^{2}, 0$ and 1 . We therefore define variance differences

$$
\Delta \sigma_{n /}^{2} \equiv \frac{\overline{(n-\bar{n})^{2}}}{\bar{n}}-1 \text { and } \Delta \sigma_{p_{t}: n}^{2} \equiv \frac{\overline{\left(p_{t}-n \hat{p}_{t}\right)^{2}}}{\bar{n}}-\sigma_{\hat{p}_{t}}^{2}=\frac{\overline{\sum_{i \neq j=1}^{n, n-1}\left(p_{t i}-\hat{p}_{t}\right)\left(p_{t j}-\hat{p}_{t}\right)}}{\bar{n}}
$$

Those relations explain the omission of $\sigma_{\hat{p}_{t}}^{2}$ in the denominator of the normalized $p_{t}: n$ variance; we want to insure measure compatibility for $p_{t}$ and $n$ fluctuations and correlations. $p_{t}$ fluctuation measure $\Phi_{p_{t}}=\sqrt{\overline{\left(p_{t}-n \hat{p}_{t}\right)^{2}} / \bar{n}}-\sqrt{\sigma_{\hat{p}_{t}}^{2}}$ [3] is the r.m.s. version of $\Delta \sigma_{p_{t}: n}^{2}$. The variance differences are per-particle fluctuation measures directly related to angular autocorrelations, as discussed in the next section.

These definitions combine the merits of Pearson's normalized covariance, tests of linear superposition and the scale-invariance of the total variance as a manifestation of the central limit theorem with a self-consistent treatment of $p_{t}$ and $n$ fluctuations and correlations.

\section{Correlations, fluctuations and inversion}

Fluctuations are directly connected to correlations by a simple relation, making the interpretation of fluctuations straightfoward. A fluctuation measurement at a single scale (e.g., the STAR TPC acceptance) is shown in the first panel of Fig. 3 [16]. The frequency histogram on $\left(p_{t}-n \hat{p}_{t}\right) /\left(\sqrt{\bar{n}} \sigma_{\hat{p}_{t}}\right)$ is compared to a central-limit reference (narrower, dashed peak). $\sigma_{\hat{p}_{t}}^{2}$ is the single-particle variance. Variance difference $\Delta \sigma_{p_{t}: n}^{2}(\delta x)$ defined above quantifies the variance excess. Questions then arise how to interpret the fluctuation measurement and how to compare it to measurements made with other detectors.
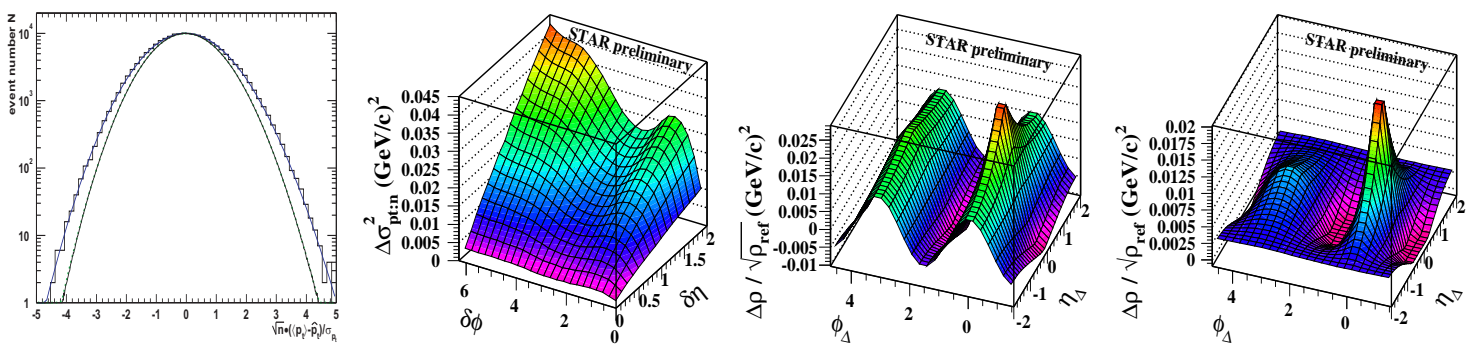

Figure 3: $\left\langle p_{t}\right\rangle$ fluctuations measured at the STAR detector acceptance (histogram) compared to a centrallimit reference (dotted curve), the scale dependence of $\left\langle p_{t}\right\rangle$ fluctuations within the STAR acceptance and the corresponding $p_{t}$ autocorrelation obtained by inversion.

Fluctuation measurements in different scale intervals explore different parts of a common distribution of fluctuation scale dependence [17], as shown in the second panel of Fig. 3. The variance difference from the first panel corresponds to the single point at the apex of the surface in the second panel. The surface is structured, but what does the structure mean? Fluctuation scale dependence is the running integral of an autocorrelation. The corresponding integral equation is a linear relation between a variance difference and an autocorrelation, including a kernel representing the binning 
scheme. We equate the per-particle variance difference on scales $(\delta \eta, \delta \phi)$ to a $2 \mathrm{D}$ running integral

$$
\Delta \sigma_{p_{t}: n}^{2}(\delta \eta, \delta \phi)=4 \int_{0}^{\delta \eta} d \eta_{\Delta} \int_{0}^{\delta \phi} d \phi_{\Delta} K\left(\delta \eta, \delta \phi ; \eta_{\Delta}, \phi_{\Delta}\right) \frac{\Delta \rho}{\sqrt{\rho_{r e f}}}\left(\eta_{\Delta}, \phi_{\Delta}\right) .
$$

The discrete form is

$$
\Delta \sigma_{p_{t}: n}^{2}\left(m \varepsilon_{\eta}, n \varepsilon_{\phi}\right)=4 \sum_{k, l=1}^{m, n} \varepsilon_{\eta} \varepsilon_{\phi} K_{m n ; k l} \frac{\Delta \rho\left(p_{t}: n ; k \varepsilon_{\eta}, l \varepsilon_{\phi}\right)}{\sqrt{\rho_{r e f}\left(n ; k \varepsilon_{\eta}, l \varepsilon_{\phi}\right)}},
$$

with kernel $K_{m n ; k l} \equiv(m-k+1 / 2) / m \cdot(n-l+1 / 2) / n$ representing the 2D macrobin system (cf. Fig. 5, right panels). The integral equation can be inverted (solved) by standard numerical methods to obtain normalized covariance density $\Delta \rho / \sqrt{\rho_{\text {ref }}}$ as an autocorrelation on difference axes $\left(\eta_{\Delta}, \phi_{\Delta}\right)$ [2]. The third panel of Fig. 3 shows the $p_{t}$ angular autocorrelation corresponding to the fluctuation data in the second panel, with directly interpretable structure: elliptic flow and minijets [5]. A similar analysis has been applied to the Hijing Monte Carlo [17]. The fourth panel shows the result of subtracting the elliptic flow contribution $\propto \cos \left(2 \phi_{\Delta}\right)$ to reveal the details of minijet correlations and illustrates how differential the autocorrelation method is. From Fig. 3 we see that fluctuation inversion provides a Rosetta stone for fluctuation and correlation analysis

\section{Comparing autocorrelations from pair counting and inversion}

Autocorrelations in the form $A(\tau) \equiv 1 / T \int_{-T / 2}^{T / 2} f(t) f(t+\tau) d t$ were first developed for timeseries analysis [1]. Time-series autocorrelations are most useful when function $f(t)$ is stationary: its correlation structure does not depend on absolute time. The structure of $f(t)$ is then completely represented statistically by the autocorrelation on the difference axis (lag) $\tau$. Autocorrelation analysis can also be applied to spatial correlations. If event-wise structures are randomly positioned on $x$ then the corresponding ensemble-average two-point distribution on $\left(x_{1}, x_{2}\right)$ is stationary (does not depend on absolute position).
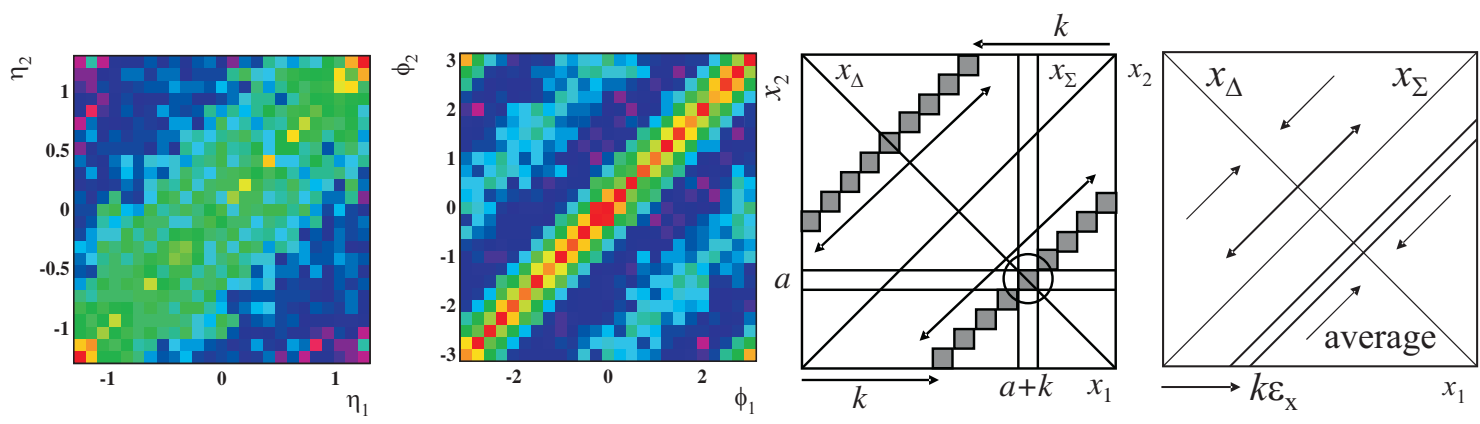

Figure 4: Two-particle correlations on $\eta$ and $\phi$ for central $\mathrm{Au}-\mathrm{Au}$ collisions at $130 \mathrm{GeV}$, schematic of a binned two-particle space illustrating an autocorrelation average along the $k^{\text {th }}$ diagonal and a similar averaging procedure performed directly on difference variable $x_{\Delta}$.

Fig. 4 (left panels) shows distributions of pair density ratios $\rho\left(x_{1}, x_{2}\right)_{\text {object }} / \rho\left(x_{1}, x_{2}\right)_{\text {reference }}$ on $\eta$ and $\phi$ typical of Au-Au collisions at RHIC [18]. The distributions are stationary-independent of position on sum axis $x_{\Sigma} \equiv x_{1}+x_{2}$ (mean pair position). All structure appears on difference axis 
$x_{\Delta} \equiv x_{1}-x_{2}$. Stationarity on $\eta$ and $\phi$ implies that the joint or 2D angular autocorrelation on $\left(\eta_{\Delta}, \phi_{\Delta}\right)$ is a lossless compression of the $4 \mathrm{D}$ two-particle angle space to the $2 \mathrm{D}$ difference-axis space. We average the two-particle density on $x_{\Sigma}\left[\rho\left(x_{\Sigma}, x_{\Delta}\right) \rightarrow \rho\left(x_{\Delta}\right)\right]$ and obtain the autocorrelation density on $x_{\Delta}$ (still a 2D density, not a projection).

Fig. 4 (right panels) illustrates two methods of autocorrelation construction. We can bin 1D space $x$ with microbins of size $\varepsilon_{x}$ and average the resulting 2D bin contents on $\left(x_{1}, x_{2}\right)$ along diagonals (third panel) or we can bin difference variable $x_{\Delta}$ on space $\left(x_{1}, x_{2}\right)$ and form the corresponding autocorrelation average directly (fourth panel) [2]. If the second procedure is applied simultaneously to $(\eta, \phi)$ we obtain $2 \mathrm{D}$ angular autocorrelations on $\left(\eta_{\Delta}, \phi_{\Delta}\right)$. Examples from data for number $n$ and $p_{t}$ angular autocorrelations are shown respectively in the left panels of Fig. 5. They are qualitatively similar (minijet structure), but there are important quantitative and physics-related differences.
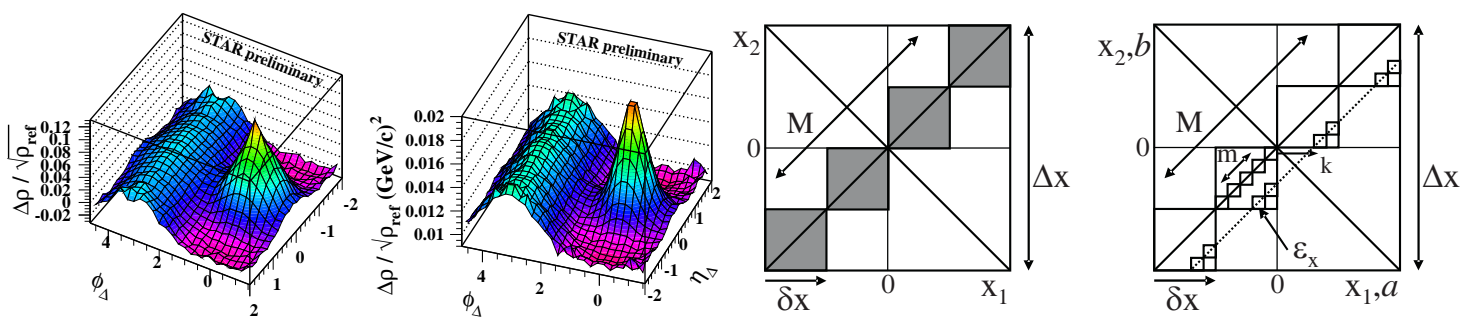

Figure 5: Left panels: Angular autocorrelations for $n$ and $p_{t}$ respectively. Right panels: Macrobins of scale $\delta x$ on a two-particle space after binning a primary space $x$, microbins of size $\varepsilon_{x}$ relative to macrobins and two microbin schemes, one on $x$, the other on $x_{\Delta}$, showing the relationships underlying the integral equation.

Fig. 5 (right panels) shows the relation between fluctuation scale dependence and autocorrelations, the origin of kernel $\mathrm{K}$ in Eq. (6.2). Autocorrelations can be obtained directly by pair counting, as in Fig. 5 (left panels) or indirectly by inversion of fluctuation scale dependence. The third panel shows a macrobin average at scale $\delta x$ over the detector acceptance $\Delta x$. The fourth panel shows a microbin average. The first is the integral of the second. The kernel $\mathrm{K}$ is determined by the geometrical relationship between the two bin systems [2]. The agreement between pair counting and fluctuation inversion is excellent for typical RHIC data (agreement at the percent level). Pair counting provides direct access to autocorrelations, but is a computationally expensive $O\left(n^{2}\right)$ process. Fluctuation inversion is a computationally cheap $O(n)$ process. Inversion of fluctuation scaling typically provides immediate physical interpretation of fluctuations and saves a factor $10 \times$ in computation time for minimum-bias Au-Au collisions at $200 \mathrm{GeV}$.

\section{Undistorted projection of 6D two-particle momentum space to 2D subspaces}

Given the correspondence between fluctuation measurements and two-particle correlations one can ask what is the overarching goal of correlation measurement, and what is the optimal measurement system? All information in the final-state momentum space $\left(y_{t}, \eta, \phi\right)$ is contained in the set of multiparticle spaces, of which the single- and two-particle spaces are computationally accessible with reasonable cost. The two-particle space is $6 \mathrm{D}$, so the correlation analysis problem becomes how to project the $6 \mathrm{D}$ space to visualizable 2D subspaces with minimum information loss or distortion and maximum interpretability. 

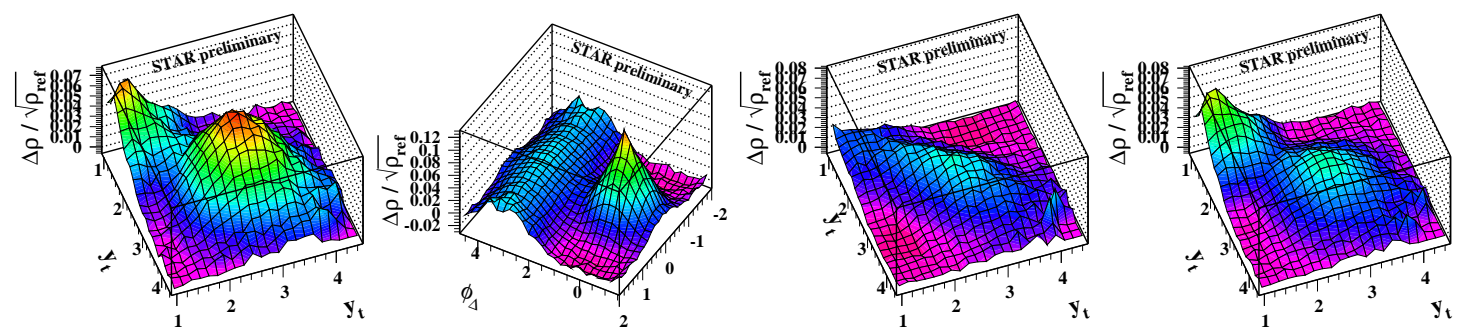

Figure 6: Efficient projection of two-particle space $\left(y_{t 1}, \eta_{1}, \phi_{1} ; y_{t 2}, \eta_{2}, \phi_{2}\right)$ to $2 \mathrm{D}$ subspaces: $\left(y_{t 1}, y_{t 2}\right)$ for all particles showing soft and hard components, joint angular autocorrelation on $\left(\eta_{\Delta}, \phi_{\Delta}\right)$ from hard component in first panel showing same-side $\left(\phi_{\Delta}<\pi / 2\right)$ and away-side $\left(\phi_{\Delta}>\pi / 2\right)$ components, corresponding distributions on $\left(y_{t 1}, y_{t 2}\right)$ for same-side and away-side components respectively.

Fig. $6[19,20]$ illustrates an optimal projection strategy. The first panel shows subspace $\left(y_{t 1}, y_{t 2}\right)$ for all pairs in minimum-bias $200 \mathrm{GeV}$ p-p collisions: all the information from number correlations on transverse rapidity $y_{t}$ for $p_{t} \in[0.15,6] \mathrm{GeV} / \mathrm{c}$. The second panel shows the complementary angular subspace, the angular number autocorrelation, with a cut in the first panel $\left(y_{t}>2\right.$, $\left.p_{t}>0.5 \mathrm{GeV} / \mathrm{c}\right)$ to isolate the hard component. That subspace reveals minimum-bias jet correlations (transverse parton fragmentation-minijets). A second angular autocorrelation (not shown) corresponds to the soft component in the first panel with $y_{t}<2$, revealing longitudinal fragmentation. Each angular autocorrelation has a same-side (SS, $\left.\phi_{\Delta}<\pi / 2\right)$ and away-side (AS, $\left.\phi_{\Delta}>\pi / 2\right)$ region.

With a cut on $\phi_{\Delta}$ we can in turn decompose the $\left(y_{t 1}, y_{t 2}\right)$ subspace (first panel) into SS and AS parts, as shown in the right two panels. The hard components are intra-jet (third panel) and inter-jet (fourth panel) number correlations (for unlike-sign or US pairs). To summarize, beyond the projection onto rapidity and angle subspaces the different pair categories are hard and soft on $\left(y_{t 1}, y_{t 2}\right)$, SS and AS on $\left(\eta_{\Delta}, \phi_{\Delta}\right)$ and LS and US in either case. That decomposition distinguishes all the details of soft hadronization and jet phenomenology in p-p collisions, with no bias from a trigger-particle condition as in conventional high- $p_{t}$ analysis [21].

\section{Two correlation types}

We identify two correlation types based on what physical mechanism produces 'correlated' pairs. As noted, correlations reflect event-wise variations in the single-particle momentum distribution. Broadly speaking, such variations arise because of transport of number or $p_{t}$ from place to place in momentum space relative to a mean-value reference, or because additional particle number or $p_{t}$ appears infrequently in some special events. Both correlation types may appear in the same data.

Fig. 7 illustrates the two correlation types. In the first panel is the minimum-bias distribution on $\left(y_{t}, y_{t}\right)$ from $200 \mathrm{GeV}$ p-p collisions, an example of correlations due to special events [19, 20]. In about $1 \%$ of non-single-diffractive (NSD) collisions (hard p-p collisions) detectable parton scattering occurs producing additional particles localized on $y_{t}$ [22]. The two-particle distribution for unexceptional (soft) collisions forms the reference. The second panel sketches contours on a 2D event frequency distribution for a pair of bins $(a, b)$ on $y_{t}$. For ordinary events (the soft reference) 

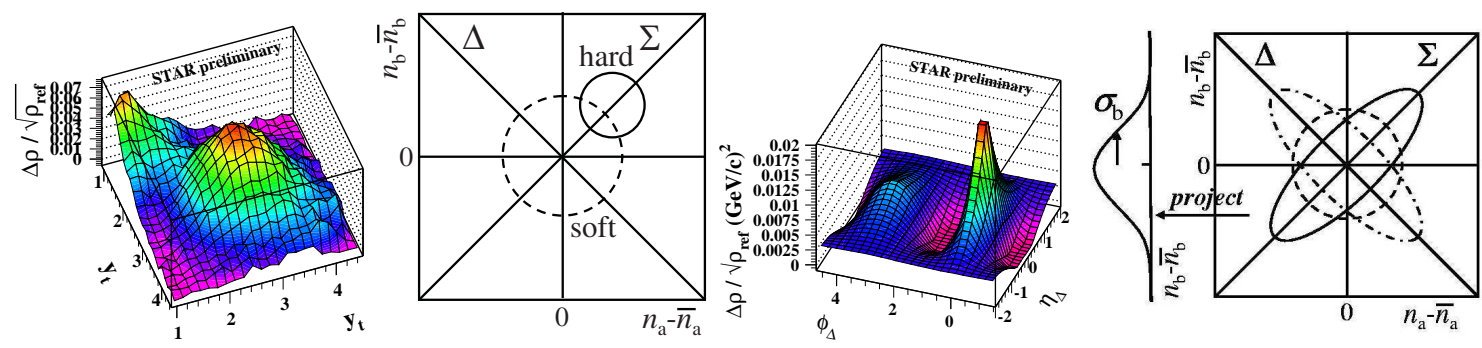

Figure 7: $p_{t}$ autocorrelation with regions of positive and negative covariance, distributions of bin-pair contents illustrating corresponding correlation (solid) and anticorrelation (dash-dot) trends, distribution on $\left(y_{t 1}, y_{t 2}\right)$ illustrating soft and hard components, and distributions of bin-pair elements illustrating the role of rare hard events in producing positive-definite covariance.

bin pairs are uncorrelated (dashed contour). However, in some events certain pairs of $y_{t}$ bins contain additional particles (solid contour). The positive covariance relative to the reference appears, when averaged over an event ensemble, as an elevated histogram bin in the first panel. In principle there are no negative bins in such a distribution (except Poisson fluctuations).

The third panel shows a $p_{t}$ angular autocorrelation for $200 \mathrm{GeV}$ mid-central Au-Au collisions obtained from $\left\langle p_{t}\right\rangle$ fluctuation inversion [5]. A flow sinusoid has been subtracted. In those collisions each event contains many (10-20) minijets. There are no special events. The correlation issue is number and $p_{t}$ transport on angle relative to an ensemble-mean distribution. The angular autocorrelation contains positive and negative regions. The fourth panel illustrates the bipolar range of covariances between bin pairs. The same-side peak at the angular origin corresponds to the solid ellipse and positive covariance. The negative regions adjacent to it on $\phi_{\Delta}$ correspond to the dash-dot ellipse describing negative covariance or anticorrelation. The mixed-pair reference is represented by the dashed circle.

\section{The physics behind correlations and fluctuations}

We summarize the physical mechanisms currently believed to produce observed fluctuations and correlations in RHIC p-p and Au-Au collisions. Different mechanisms are inferred by separating correlation data according to $y_{t}$ (soft, hard), $\phi_{\Delta}$ (SS, AS) and charge-pair type (LS, US). The differential correlation structure is then typically suggestive of the underlying dynamics.

\subsection{The physics of $n$ fluctuations}

Each bin pair from the single-particle momentum space is characterized by a frequency distribution as sketched in Fig. 8 (first panel), where particle charge is also a label. Fluctuations in the yield of positive particles in one bin is compared with fluctuations of negative particles in another bin. The difference between variances on the sum $n_{c h}$ and difference $Q$ axes correspond to a +covariance. Covariance distributions for LS and US pair types are also combined as CI $=\mathrm{LS}+\mathrm{US}$ and $\mathrm{CD}=\mathrm{LS}-\mathrm{US}$ to obtain isoscalar (CI) [23] and isovector (CD) [18] correlations respectively. For bins $a, b$ of size $\varepsilon_{x}$ on variable $x$ the normalized number covariance density is

$$
\frac{\Delta \rho}{\sqrt{\rho_{r e f}}} \equiv \frac{\overline{(n-\bar{n})_{a}(n-\bar{n})_{b}}}{\varepsilon_{x} \sqrt{\bar{n}_{a} \bar{n}_{b}}}
$$



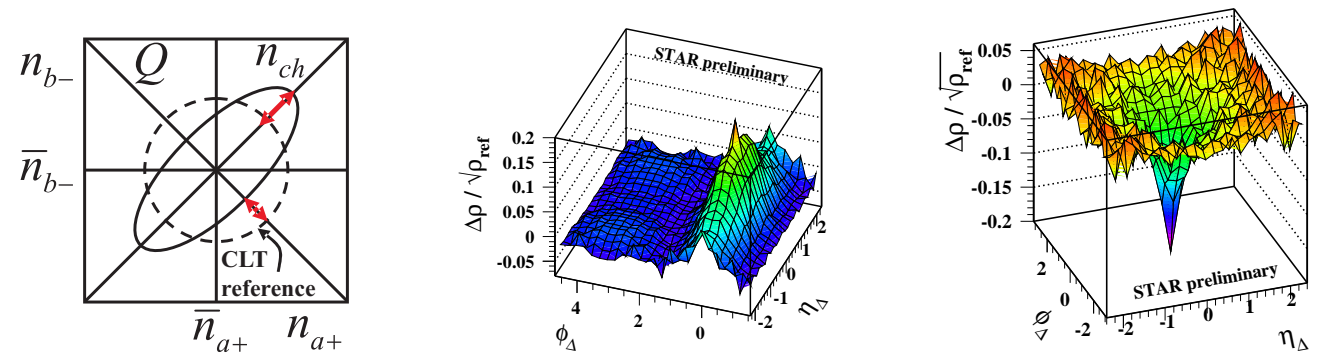

Figure 8: Illustration of physics processes revealed by multiplicity fluctuations. Replace third panel by one with elliptic flow. Note that there is no flow in fourth panel.

In Fig. 8 (right panels) we show CI and CD number angular autocorrelations from $130 \mathrm{GeV}$ $\mathrm{Au}-\mathrm{Au}$ collisions obtained by pair counting $[18,23]$. The three principal physics mechanisms for CI correlations are longitudinal ('string') fragmentation, elliptic flow and transverse (parton) fragmentation (minijets). The second panel shows the CI number autocorrelation for mid-central $\mathrm{Au}-\mathrm{Au}$ collisions with the elliptic flow sinusoid subtracted. The same-side minijet peak is strongly elongated on $\eta$ [23]. There is no away-side jet ridge (previously established by trigger-particle studies [21]) and no soft or longitudinal fragmentation correlation, (gaussian on $\eta_{\Delta}$ ). The minijet deformation and disappearance of the soft component [23] are new observations established with minimum-bias angular autocorrelations [2].

The third panel shows the $\mathrm{CD}$ number autocorrelation for mid-peripheral $\mathrm{Au}-\mathrm{Au}$ collisions. There is no elliptic flow structure observed in CD correlations (another new observation [18]). We observe a large-amplitude negative peak, symmetric about the angular origin with nearly exponential shape. The form of the CD peak is very different from the CI minijet peak and from the comparable CD structure in p-p collisions $[19,20]$. Whereas the CI minijet peak in mid-central $\mathrm{Au}-\mathrm{Au}$ is well-described by a $2 \mathrm{D}$ gaussian strongly elongated on $\eta$, the $\mathrm{CD}$ peak is a symmetric exponential. Its shape and other properties suggest that the $\mathrm{CD}$ structure arises from 2D surface hadronization from the A-A medium. Subsequent hadronic rescattering attenuates the correlation structure with increasing pair opening angle, producing the sharp exponential fall-off [18].

\subsection{The physics of $p_{t}$ fluctuations}

Although there are underlying commonalities between $p_{t}$ and $n$ fluctuations and correlations (minijets and elliptic flow produce qualitatively similar structures in both) there are important differences in detail. Number angular correlations can only reveal the particle flux in a given angular region, whereas $p_{t}$ correlations can reveal the velocity and/or temperature of the local particle source. The dominant theme of $p_{t}$ angular correlations is particle emission from locally-moving sources. The source velocity structure can be local (minijets) or extend over a broad angular region (elliptic flow). For microbins $a, b$ of size $\varepsilon_{x}$ on angular variable $x$ the normalized $p_{t}$ covariance density is

$$
\frac{\Delta \rho}{\sqrt{\rho_{\text {ref }}}}=\frac{\overline{\left(p_{t}-n \hat{p}_{t}\right)_{a}\left(p_{t}-n \hat{p}_{t}\right)_{b}}}{\varepsilon_{x} \sqrt{\bar{n}_{a} \bar{n}_{b}}}
$$

In Fig. 9 (first panel) we illustrate the problem of distinguishing among 1) $p_{t}$ fluctuations relative to particle number fluctuations, 2) fluctuations of particle number relative to a fixed reference, 

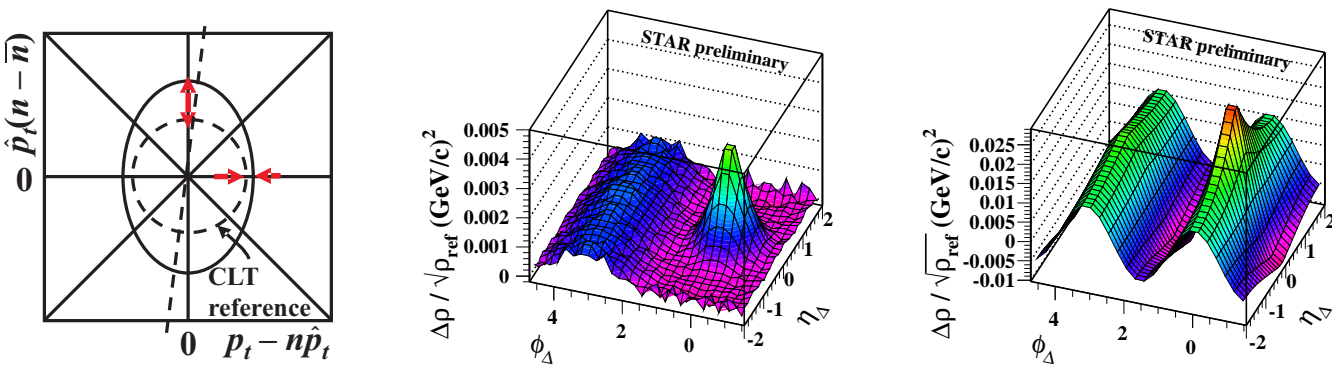

Figure 9: Illustration of physics processes revealed by $p_{t}$ fluctuations.

and 3) the covariance between the two types of fluctuations. Decomposition of the $p_{t}$ variance to three terms was presented in Eq. (5.3) [13]. The first term on the RHS of that equation corresponds to $\left(p_{t}-n \hat{p}_{t}\right)$ fluctuations on the $\mathrm{x}$ axis of the first panel. The third term corresponds to $(n-\bar{n})$ fluctuations on the y axis. The second term in Eq. (5.3) corresponds to a possible rotation of the 2D frequency distribution in the first panel illustrated by the dashed line and signaling a nonzero $p_{t} \cdot n$ covariance. The central-limit reference is the dashed circle. $p_{t}$ fluctuations and correlations are not trivially related to number fluctuations and correlations. Careful distinctions are required, and failure to achieve those distinctions in statistical measure design results in biases, including punchthrough of number correlations into inferred $p_{t}$ correlations.

In the right two panels we show $p_{t}$ angular autocorrelations from $200 \mathrm{GeV}$ p-p (second panel) and $\mathrm{Au}-\mathrm{Au}$ (third panel) collisions. The second panel was obtained by pair counting, the third by fluctuation inversion. The p-p data show a standard same-side jet cone and an away-side ridge. The shape of the same-side peak is considerably different for $p_{t}$ correlations, approaching an exponential peak compared to a gaussian for number correlations from unbiased partons. The reason is simple: larger- $p_{t}$ fragments tend to appear at smaller angles relative to the jet thrust axis, a consequence of the parton fragmentation process. Those correlations were obtained with no trigger particle. They are dominated by partons with $Q \sim 4 \mathrm{GeV}$ fragmenting to two hadrons (pions) with US charge combination and most-probable $p_{t} \sim 1 \mathrm{GeV} / \mathrm{c}$. They provide unique new information on the most prolific manifestation of QCD processes in nuclear collisions at RHIC.

The Au-Au data in the third panel are dominated by elliptic flow (sinusoid) and a same-side jet peak strongly elongated on pseudorapidity, as we saw for number correlations. However, a new feature unique to $p_{t}$ correlations is the pair of depressions on either side of the jet peak on $\phi_{\Delta}$. Detailed analysis suggests that those depressions are part of a broad negative peak under the positive same-side peak [5]. A possible interpretation of the new feature is medium recoil from stopping the inward-going parton partner of the parton fragmenting to the positive same-side peak. The recoil produces a red shift of the local $p_{t}$ spectrum. In essence, the positive and negative peaks reflect local momentum conservation in the radial direction during parton scattering.

\section{Comments on measure design}

One can illustrate good measure design by contrast with design misconceptions. We consider some measures which do not provide an intuitive indication of the underlying physical mechanisms for correlation trends on collision centrality and energy. 


\section{1 $\sigma_{\left\langle p_{t}\right\rangle}^{2}, \sigma_{p_{t}, \text { dynamical }}^{2}$ and measure bias}

Measure bias results from placing random variables in denominators. As an example we consider the bias in $\sigma_{\left\langle p_{t}\right\rangle}^{2} \equiv \overline{\left\{\left(p_{t}-n \hat{p}_{t}\right)^{2} / n^{2}\right\}}$. We switch to $\bar{n} \sigma_{\left\langle p_{t}\right\rangle}^{2}$ to make a comparison below.

$$
\bar{n} \sigma_{\left\langle p_{t}\right\rangle}^{2} \equiv \overline{\left\{\frac{\left(p_{t}-n \hat{p}_{t}\right)^{2}}{\bar{n}} \cdot \frac{\bar{n}^{2}}{n^{2}}\right\}} \text { with } \frac{\bar{n}^{2}}{n^{2}}=1-2 \frac{\delta n}{\bar{n}}+3 \frac{(\delta n)^{2}}{\bar{n}^{2}}+\cdots
$$

We incorporate the Taylor expansion and form the differential fluctuation measure

$$
\bar{n} \sigma_{\left\langle p_{t}\right\rangle}^{2}-\sigma_{\hat{p}_{t}}^{2}=\Delta \sigma_{p_{t}: n}^{2}\left\{1+3 \frac{\sigma_{n}^{2}}{\bar{n}^{2}}+\cdots\right\}-2 \overline{\left.\frac{\left(p_{t}-n \hat{p}_{t}\right)^{2}}{\bar{n}} \cdot \frac{\delta n}{\bar{n}}\right\}}+3 \sigma_{\hat{p}_{t}}^{2} \frac{\sigma_{n}^{2}}{\bar{n}^{2}}+\cdots
$$

Recall that $\sigma_{n}^{2} / \bar{n} \sim 1-2$ and $\sigma_{\hat{p}_{t}}^{2} \sim 0.1(\mathrm{GeV} / \mathrm{c})^{2}$ for RHIC Au-Au collisions [16]. Referring to Fig. 3 (second panel), at a scale where $\bar{n} \sim 10$ we find that $\Delta \sigma_{p_{t}: n}^{2} \sim 0.01$, but the positive bias term on the far right is $0.03-0.06$. Thus, the third term is a catastrophic bias source for this fluctuation measure. The second term is negative because the $p_{t} \cdot n$ covariance in brackets is strongly positive: minijets produce more $p_{t}$ and more multiplicity together and localized on $(\eta, \phi)$. Eq. (11.2) is $\bar{n} \sigma_{p_{t}, \text { dynamical-I }}^{2}[6]$, which is therefore a strongly biased fluctuation measure. One could argue, reversing the equation, that $\Delta \sigma_{p_{t}: n}^{2}$ is the biased measure, but that measure has been compared to angular autocorrelations determined directly by pair counting and agrees precisely $(\sim 1 \%)$ to arbitrarily small scale [2].

\section{2 $\left\langle\delta p_{t} \cdot \delta p_{t}\right\rangle, \Sigma_{p_{t}}$ and the energy dependence of $p_{t}$ fluctuations}

$\left\langle\delta p_{t} \cdot \delta p_{t}\right\rangle \equiv \overline{\sum_{i \neq j}\left(p_{t i}-\hat{p}_{t}\right)\left(p_{t j}-\hat{p}_{t}\right) / n(n-1)}$, also denoted $\sigma_{p_{t}, \text { dynamical }}^{2}$ [8], is another of the $\left\langle p_{t}\right\rangle$ fluctuation measures introduced in Sec. 2. By analogy with the previous subsection we compare $\left\langle\delta p_{t} \cdot \delta p_{t}\right\rangle$ to $\Delta \sigma_{p_{t}: n}^{2} / \bar{n} \equiv \overline{\sum_{i \neq j}\left(p_{t i}-\hat{p}_{t}\right)\left(p_{t j}-\hat{p}_{t}\right)} / \bar{n}^{2}$. The differences are two-fold: 1$)$ there is an additional factor $n$ in the denominator of $\left\langle\delta p_{t} \cdot \delta p_{t}\right\rangle$, and 2) $\Delta \sigma_{p_{t}: n}^{2}$ is a ratio of ensemble averages while $\left\langle\delta p_{t} \cdot \delta p_{t}\right\rangle$ an ensemble average of a ratio of random variables. The extraneous factor $n$ in the denominator of $\left\langle\delta p_{t} \cdot \delta p_{t}\right\rangle$ produces misleading centrality and energy trends, a problem shared with net-charge fluctuation measure $v_{\text {dynamical }}$ also previously discussed. Ratios of random variables produce bias terms by construction. A Taylor-expansion of the denominator of $\left\langle\delta p_{t} \cdot \delta p_{t}\right\rangle$ reveals that the large positive bias term in the original $\sigma_{p_{t}, \text { dynamical }}^{2}$ is eliminated in the newer version, leaving the negative covariance term as the main bias source.

The definitions and interpretation of $\Sigma_{p_{t}} \equiv \sqrt{\left\langle\delta p_{t} \cdot \delta p_{t}\right\rangle} / \hat{p}_{t}[8,9]$ are based on an assumption of global event-wise thermalization. If true, $\left\langle p_{t}\right\rangle$ fluctuations are caused only by $T$ fluctuations. $\left\langle\delta p_{t} \cdot \delta p_{t}\right\rangle$ then estimates temperature variance $\sigma_{T}^{2}, \hat{p}_{t}$ estimates mean temperature $T_{0}$, and $\Sigma_{p_{t}}$ therefore estimates the r.m.s. relative variation $\delta T / T_{0}$. Given those assumptions it is claimed that $\delta T / T_{0}$ (and therefore $\left\langle p_{t}\right\rangle$ fluctuations) has no significant collision energy dependence based on measurements of $\Sigma_{p_{t}}$. However, when $\Delta \sigma_{p_{t}: n}^{2}, \hat{p}_{t}$ and $\bar{n}$, are examined independently they reveal clear evidence that the thermalization assumption is wrong [10]. Events are highly structured. $\Delta \sigma_{p_{t}: n}^{2}$ is dominated by minijets, as revealed by model-independent fluctuation/correlation analysis [5]. The energy dependence is strong, and consistent with QCD systematics. The a priori imposition of a model on measure design in this example yields a misleading result. 


\subsection{Forward-backward correlations}

Forward-backward (FB) correlations describes an early form of correlation analysis on rapidity or pseudorapidity applied to elementary collisions at lower energies [15]. The binning scheme is shown in Fig. 2 (fourth panel). The measure is $F B \equiv \frac{\sigma_{f b}^{2}}{\sigma_{f}^{2}} \rightarrow \frac{\sigma_{f b}^{2}}{\sqrt{\sigma_{f}^{2} \cdot \sigma_{b}^{2}}}$ (by symmetry about the $\mathrm{CM}$ ), exactly Pearson's normalized covariance between two symmetric rapidity bins. $F B$ is simply related to the CI normalized covariance density by $\Delta \rho /{\sqrt{\rho_{r e f}}}_{a b} \equiv \frac{\sigma_{a b}^{2}}{\varepsilon_{\eta} \sqrt{\bar{n}_{a} \cdot \bar{n}_{b}}}=\frac{\sigma_{a b}^{2}}{\varepsilon_{\eta} \sqrt{\sigma_{a}^{2} \cdot \sigma_{b}^{2}} C L T} \rightarrow$ $\frac{\sigma_{a b}^{2}}{\sqrt{\sigma_{a}^{2} \cdot \sigma_{b}^{2}}}=F B$ if $(a, b) \rightarrow(f, b)$. FB is thus (within an $O(1)$ factor) the autocorrelation on $\eta_{\Delta}$ projected from the 2D number autocorrelation on $\left(\eta_{\Delta}, \phi_{\Delta}\right)$.

Recent applications of $F B$ invoke the historical context in attempting to identify SRC and LRC components (short- and long-range correlations) in terms of string-fragmentation and the dualparton model [15]. There are several problems with that approach: 1) the historical measure is not properly related to the modern analysis context; 2) the terms SRC, LRC are not defined in terms of presently-understood physical processes; 3 ) the theory-inspired analysis procedures used to isolate SRC and LRC components are poorly justified, especially in light of more recent progress.

\subsection{The balance function}

Like the FB, the original balance function (BF1) was applied to elementary collisions at lower energies to study local measure conservation $(\mathrm{Q}, \mathrm{S}, \mathrm{B})$ at the beginning and end of the e-e or $\mathrm{p}-\mathrm{p}$ collision process [24]. BF1 was a true conditional distribution: a projection from two-particle space $\left(y_{1}, y_{2}\right)$ onto one rapidity axis given a condition (bin) defined on the other. Significant correlation structure deviated from a uniform background. BF1 was instrumental in the development of QCD theory. The second version BF2 is fundamentally different, although the same name and similar algebra are invoked [25]. BF2 is a projection onto diagonal difference axis $y_{1}-y_{2}$ or $\eta_{1}-\eta_{2}$. The constant offset in the original version becomes an acceptance triangle, and true net-charge correlations vary about that triangle. The reference triangle dominates the overall structure, presenting a misleading picture.

The physics of BF2 relates to its width. BF2 theory [25] argues that charge diffusion during hadronic rescattering should increase the net-charge correlation length on rapidity. The net-charge correlation length is estimated (according to [25]) by the BF2 width. Thus, reduction of the BF2 width with centrality would indicate reduced diffusion of hadronic charge, or 'late hadronization.' However, the width of BF2 is dominated by the reference triangle. For typical nuclear collisions the composite $\mathrm{BF} 2$ width is completely insensitive to the correlation length of local net-charge correlations $[18,26]$. The BF2 width variation is dominated by changes in the amplitude of netcharge correlations, leading to incorrect inferences. BF2 is simply related to the CD (net-charge) angular autocorrelation, which has been measured for $130 \mathrm{GeV} \mathrm{Au}-\mathrm{Au}$ collisions [18] and gives a very different picture of local charge conservation than the theoretical hypothesis which motivated BF2. A third variant, $B F(\infty) \rightarrow B F 3$, has been defined with an approximation to the acceptance factor removed [27]. $B F 3$ is therefore approximately a projection of the 2D net-charge angular autocorrelation onto the $\eta_{\Delta}$ axis. The confusion imposed by $B F 2$ and $B F 3$ has essentially halted progress on the physics of net-charge correlations. 


\section{Summary}

We have reviewed the current status of fluctuation and correlation analysis methods at the SPS and RHIC. We have identified some basic issues of measure design, and shown how they can be used to define scale-dependent differential fluctuation measures for number and $p_{t}$ fluctuations. We show that fluctuation scale dependence is related to angular autocorrelations by an integral equation. Autocorrelations from fluctuation inversion and pair counting are comparable at the percent level, and the former, being much faster, saves a great deal of computing time for heavy ion collisions. Given the connection between fluctuations and correlations we discuss the optimum projection of two-particle momentum space to $2 \mathrm{D}$ subspaces with minimal distortion. We consider two general correlation types and describe their manifestations in collision data. We then list the currently-known mechanisms which produce number and $p_{t}$ correlations. We conclude with comments on several measure designs which illustrate the need for good design principles.

This work was supported in part by the Office of Science of the U.S. DoE under grant DEFG03-97ER41020.

\section{References}

[1] G. E. P. Box and G. Jenkie, Time series analysis: forecasting and control, Holden-Day (1976); J. S. Bendat and A. G. Persol, Random data - analysis and measurement procedures, $2^{\text {nd }}$ Edition, John Wiley \& Sons, NY (1986).

[2] T. A. Trainor, R. J. Porter and D. J. Prindle, J. Phys. G: Nucl. Part. Phys. 31, 809 (2005).

[3] M. Gazdzicki and S. Mrowczynski, Z. Phys. C 54 (1992) 127.

[4] H. Appelshauser et al. (NA49 Collaboration), Phys. Lett. B 459, 679 (1999).

[5] J. Adams et al. (STAR Collaboration), J. Phys. G 32, L37 (2006).

[6] S. A. Voloshin, V. Koch and H. G. Ritter, Phys. Rev. C 60, 024901 (1999).

[7] S. S. Adler et al. (PHENIX Collaboration), Phys. Rev. Lett. 93, 092301 (2004).

[8] J. Adams et al. (STAR Collaboration), Phys. Rev. C 72, 044902 (2005).

[9] D. Adamova et al. (CERES Collaboration), Nucl. Phys. A 727, 97 (2003).

[10] J. Adams et al. (STAR Collaboration), nucl-ex/0605021.

[11] S. Jeon and V. Koch, Phys. Rev. Lett. 85, 2076 (2000).

[12] J. Adams et al. (STAR Collaboration), Phys. Rev. C 68, 044905 (2003).

[13] T. A. Trainor, hep-ph/0001148

[14] K. Pearson, Phil. Trans. Royal Soc. 187, 253 (1896).

[15] A. Capella, and A. Krzywicki, Phys. Rev. D 184, 120 (1978).

[16] J. Adams et al. (STAR Collaboration), Phys. Rev. C 71, 064906 (2005).

[17] Q. J. Liu, D. J. Prindle and T. A. Trainor, Phys. Lett. B 632, 197 (2006).

[18] J. Adams et al. (STAR Collaboration), Phys. Lett. B 634, 347 (2006).

[19] R. J. Porter and T. A. Trainor (STAR Collaboration), Acta Phys. Polon. B 36, 353 (2005). 
[20] T. A. Trainor (STAR Collaboration), Proceedings of the International Symposium on Multiparticle Dynamics (ISMD 2005), Kromêr̂́î́, CR, August 9-15, 2005, Published in AIP Conf. Proc. 828, 238 (2006).

[21] C. Adler et al. (STAR Collaboration), Phys. Rev. Lett. 90, 082302 (2003).

[22] J. Adams et al. (STAR Collaboration), Phys. Rev. D 74, 032006 (2006).

[23] J. Adams et al. (STAR Collaboration), Phys. Rev. C 73, 064907 (2006).

[24] R. Brandelik et al., Phys. Lett. B 100357 (1981); H. Aihara et al., Phys. Rev. Lett. 532199 (1984); P.D. Acton et al., Phys. Lett. B 305415 (1993); T. Kafka et al., Phys. Rev. D 16 (1977) 1261; J. Whitmore, Phys. Repts. 27, 187-273 (1976).

[25] S. A. Bass, P. Danielewicz and S. Pratt, Phys. Rev. Lett. 85, 2689 (2000).

[26] T. A. Trainor, hep-ph/0301122.

[27] S. Jeon and S. Pratt, Phys. Rev. C 65, 044902 (2002). 